1 Instituto Estadual de Diabetes e Endocrinologia (IEDE) e Pontifícia Universidade Católica do Rio de Janeiro (PUC-RJ), Rio de Janeiro, RJ, Brasil 2 Universidade Federal do Estado do Rio de Janeiro (UNIRIO), Rio de Janeiro, RJ, Brasil

Correspondência para: Rodrigo O. Moreira Instituto Estadual de Diabetes e Endocrinologia

Rua Moncorvo Filho, 90 20211-340 - Rio de Janeiro, RJ, Brasil

rom_br@yahoo.com

Recebido em 14/Agol/2010 Aceito em 5/Jan/2011

\section{Metformina: mais uma interação medicamentosa no tratamento do hipotireoidismo?}

\author{
Metformin: one more drug interaction in the \\ treatment of hypothyroidism?
}

Rodrigo O. Moreira', Geni O. Moreira ${ }^{2}$

$\mathrm{N}^{2}$ edição dos Arquivos Brasileiros de Endocrinologia e Metabologia de julho de 2010, a Dra. Laura S. Ward apresentou uma excelente revisão sobre interação medicamentosa e o impacto de doenças concomitantes no tratamento do hipotireoidismo (1). Por ser uma doença com elevada prevalência e de tratamento relativamente simples, é necessário que todos os médicos que tratem pacientes com hipotireoidismo conheçam todos (ou a maioria) dos medicamentos que possam interferir na absorção e/ou no metabolismo da levotiroxina (LT4). Na sua revisão, a autora apresenta alguns dos medicamentos e situações mais importantes dessa interação, incluindo doenças gástricas, cirurgia bariátrica e amiodarona. Entretanto, nos últimos anos, algumas evidências vêm sendo publicadas sobre os efeitos no hipotireoidismo de um dos medicamentos mais utilizados pelos endocrinologistas para o tratamento do diabetes melito tipo 2: a metformina.

Existem, até o presente momento, três artigos publicados que sugerem que a metformina pode interferir no tratamento do hipotireoidismo. O primeiro artigo é composto por uma série de casos e foi publicado em 2006 por Vigersky e cols. (2). No manuscrito, os autores apresentam o caso de uma paciente com hipotireoidismo controlado com uma dose estável de LT4 que apresentou supressão da concentração sérica de TSH após a introdução da metformina de liberação prolongada para tratamento de esteatose hepática. A concentração plasmática do TSH retornou ao normal após a suspensão da metformina e novamente foi suprimida com o reinício da medicação. Durante todo o período, a dose da LT4 se manteve estável. Neste mesmo artigo, mais quatro pacientes são apresentados com evolução clínica semelhante: um com hipotireoidismo pós-tratamento de doença de Graves com ${ }^{131} \mathrm{I}$; um com tireoidite de Hashimoto e dois com hipotireoidismo pós-cirúrgico. Em todos os casos, houve redução da concentração sérica do TSH após a introdução da metformina, inclusive com necessidade de ajuste da dose da LT4.

Os dois artigos publicados posteriormente já envolvem um número um pouco maior de pacientes. No artigo de Isidro e cols. de 2007 (3), os autores investigam os efeitos da introdução da metformina em oito pacientes com hipotireoidismo e dose estável de LT4. Os autores observaram uma redução estatisticamente significativa da concentração plasmática do TSH após três meses de tratamento com metformina (de $3,11 \pm 0,50$ para $1,18 \pm 0,36 \mathrm{microUI} / \mathrm{ml} ; \mathrm{p}<0,05)$, com um posterior aumento do TSH três meses após a suspensão da medicação (para 2,2l $\pm 0,37 \mathrm{microUI} / \mathrm{ml}$ ). No último artigo publicado sobre o assunto por Cappelli e cols. em 2009 (4), os autores investigam dois grupos de pacientes. No primeiro grupo, os autores avaliam a variação do TSH em 11 pacientes com hipotireoidismo e dose estável de LT4 tratados por metformina por seis meses. Embora tenha sido observada apenas uma modesta e 
não significativa redução do TSH (de $2,11 \pm 0,55$ para $1,5 \pm 0,36 ; \mathrm{p}=\mathrm{NS}$ ), um dos pacientes apresentou uma supressão da concentração plasmática de TSH, que retornou ao normal após a suspensão da metformina. $\mathrm{Na}$ segunda parte do trabalho, os autores observam três resultados em três grupos de pacientes acompanhados por um ano: (1) em pacientes com hipotireoidismo adequadamente compensado com uma dose estável de LT4, houve redução significativa da concentração sérica de TSH (de 2,37 $\pm 1,17$ para 1,41 $\pm 1,21$ microUI/ $\mathrm{ml} ; \mathrm{p}<0,001) ;(2)$ em pacientes com hipotireoidismo subclínico sem tratamento, houve uma redução significativa da concentração plasmática de TSH (de 4,5 \pm $0,37$ para 2,93 $\pm 1,48 \mathrm{microUI} / \mathrm{ml} ; \mathrm{p}<0,001)$ e $(3)$ num grupo controle sem qualquer evidência de doença tireoidiana não houve diferença na concentração sérica do TSH $(2,74 \pm 0,82$ para 2,56 $\pm 1,16 ; \mathrm{p}=\mathrm{NS})$.

Não existe ainda uma explicação para esses efeitos, embora algumas hipóteses já tenham sido aventadas. Uma das explicações poderia envolver uma alteração na absorção da LT4 induzida pela metformina. Entretanto, em consequência dos conhecidos efeitos gastrointestinais da metformina, seria esperada uma diminuição da absorção da LT4, com um aumento da concentração plasmática de TSH. Além disso, essa explicação não justifica o achado de Capelli e cols. (4) da supressão do TSH em pacientes com hipotireoidismo subclínico ainda sem tratamento. Outra hipótese seria a existência de efeitos anti-inflamatórios da metformina diretamente sobre a tireoide. Entretanto, devemos lembrar que, no estudo inicial de Vigersky e cols. (2), dois pacientes apresentavam hipotireoidismo pós-cirúrgico (incluindo um com tireoidectomia total por carcinoma diferenciado da tireoide). Uma terceira explicação seria um efeito direto da metformina sobre o hipotálamo e/ou hipófise, alterando de forma significativa os mecanismos de feedback do eixo tireoidiano. Da mesma forma, poderia se especular que a redução na concentração plasmática do TSH seria secundária a uma redução do peso corporal induzida pela metformina. Em nenhum dos artigos, entretanto, foi evidenciada qualquer alteração significativa do peso que pudesse justificar essa hipótese. Finalmente, poderia existir algum efeito da metformina que comprometesse o metabolismo hepático da LT4. Essa hipótese também parece improvável, já que a metformina não sofre metabolização hepática e não foi encontrada nenhuma evidência que sugira que a metformina possa interferir na atividade das deiodinases, com a ligação da tiroxina a TBG ("Thyroxine-
-Binding Globulin") ou com o processo de degradação da tiroxina (especificamente com as reações hepáticas de sulfatação ou glicorunidação) (5). É importante ressaltar que os únicos efeitos identificáveis da metformina foram sobre a concentração sérica do TSH. Os estudos também avaliaram os outros hormônios e não foram encontradas diferenças significativas nos níveis de T3 e T4 livre, assim como da TBG.

A existência de uma possível interação da metformina sobre o tratamento do hipotireoidismo é de enorme importância clínica, assim como de diversos outros medicamentos utilizados na prática clínica (6). A relevância dessa associação baseia-se no fato de o DM tipo 2 e o hipotireoidismo serem as duas doenças mais prevalentes da endocrinologia e de frequentemente coexistirem em um mesmo paciente. Até o presente momento, não existe uma evidência concreta de que essa interação exista. Entretanto, os trabalhos acima são geradores de hipóteses, o que provavelmente levará a novos trabalhos, com mais pacientes e metodologia mais adequada, para que essa interação possa ser adequadamente investigada. Além disso, o próprio mecanismo dessa possível interação ainda permanece obscuro e precisa ser mais bem compreendido, se realmente existir. Entretanto, o endocrinologista precisa estar ciente de que tal interação pode existir e, copiando o título da revisão da Dra. Laura S. Ward (1), de que o paciente com hipotireoidismo e DM pode ser ainda mais "difícil" do que parece.

Declaração: os autores declaram não haver conflitos de interesse científico neste estudo.

\section{REFERÊNCIAS}

1. Ward LS. The difficult patient: drug interaction and the influence of concomitant diseases on the treatment of hypothyroidism. Arq Bras Endocrinol Metab. 2010;54(5):435-42.

2. Vigersky RA, Filmore-Nassar A, Glass AR. Thyrotropin suppression by metformin. J Clin Endocrinol Metab. 2006;91(1):225-7.

3. 3. Isidro ML, Penín MA, Nemiña R, Cordido F. Metformin reduces thyrotropin levels in obese, diabetic women with primary hypothyroidism on thyroxine replacement therapy. Endocrine. 2007;32(1):79-82.

4. Cappelli C, Rotondi M, Pirola I, Agosti B, Gandossi E, Valentini $U$, et al. TSH-lowering effect of metformin in type 2 diabetic patients: differences between euthyroid, untreated hypothyroid, and euthyroid on L-T4 therapy patients. Diabetes Care. 2009;32(9):1589-90.

5. Farwell AP, Braverman LE. Thyroid and antithyroid drugs. In: Goodman \& Gilman's. The pharmacological basis of therapeutics. Hardman Gilman AG, JG, Limbird LE. 9th edition. New York: McGraw-Hill; 1996.

6. Haugen BR. Drugs that suppressTSH or cause central hypothyroidism. Best Pract Res Clin Endocrinol Metab. 2009;23(6):793-800. 